\title{
A note on naturally embedded ternary trees
}

\author{
Markus Kuba* \\ Institut für Diskrete Mathematik und Geometrie \\ Technische Universität Wien \\ Wiedner Hauptstr. 8-10/104 \\ 1040 Wien, Austria \\ kuba@dmg.tuwien.ac.at
}

Submitted: Feb 3, 2009; Accepted: Jul 1, 2011; Published: Jul 15, 2011

Mathematics Subject Classification: 05A15, 05C05

\begin{abstract}
In this note we consider ternary trees naturally embedded in the plane in a deterministic way. The root has position zero, or in other words label zero, and the three children of a node with position $j \in \mathbb{Z}$ have positions $j-1, j$, and $j+1$. We derive the generating function of embedded ternary trees where all internal nodes have labels less than or equal to $j$, with $j \in \mathbb{N}$. Furthermore, we study the generating function of the number of ternary trees of size $n$ with a given number of internal nodes with label $j$. Moreover, we discuss generalizations of this counting problem to several labels at the same time. We also study a refinement of the depth of the external node of rank $s$, with $0 \leq s \leq 2 n$, by keeping track of the left, center, and right steps on the unique path from the root to the external node. The $2 n+1$ external nodes of a ternary tree are ranked from the left to the right according to an inorder traversal of the tree. Finally, we discuss generalizations of the considered enumeration problems to embedded $d$-ary trees.
\end{abstract}

Keywords: Ternary trees, Embedded trees, Labeled trees

\section{Introduction}

The study of tree families embedded in the plane has recently received a lot of attention. Binary trees, complete binary trees, families of plane trees, and more generally simply generated tree families, have been considered in a series of papers $[5,6,17,10,3,2,15$, $16,9,19,11,12]$. It has been shown that embedded trees are closely related to a random measure called ISE (Integrated SuperBrownian Excursion). For example, in the recent

*The author was partially supported by the Austrian Science Foundation FWF, grant S9608-N13. 
paper of Devroye and Janson [9] a conjecture of Bousquet-Mélou and Janson [2] is proven, saying that the vertical profile of a randomly embedded simply generated tree converges in distribution, after suitable normalization, to the density of the ISE. However, enumerative properties of deterministically embedded trees have not been intensively studied, except for binary trees [3], a particular subclass of ternary trees [13, 8], and plane trees [5, 6, 3]. The combinatorial results of $[5,6,3]$ are so far not completely understood, see [4].

Motivated by the results of Bousquet-Mélou [3] and Panholzer [19] for embedded binary trees, and the results of Schaeffer and Jacquard [13], and Del Lungo, Del Ristoro and Penaud [8] for a specific subclass of embedded ternary trees, we study several combinatorial properties of ternary trees embedded in the plane in a deterministic manner. We consider the following natural embedding. The root has position zero, or in other words label zero, and the three children of the root have positions $-1,0$ and 1 . More generally, the labels of the three children of an internal node with label $j$ are given by $j-1, j, j+1$, with $j \in \mathbb{Z}$. A similar embedding of ternary trees has been considered before $[13,8]$, where the authors studied a particular subclass of embedded ternary trees named skew ternary trees [13], or left ternary trees [8], which are embedded ternary trees with no (internal) node having label greater than zero. Using bijections between embedded ternary trees with no (internal) node of label greater than zero and non-separable rooted planar maps with $n+1$ edges they obtained amongst others an explicit result for the number of such trees of size $n$.

The aim of this work is to use a generating functions approach to study several parameters in embedded ternary trees. The depth of a node $v \in T$ is defined as the number of nodes on the unique path $P_{T}(v)$ from the root of the tree $T$ to node $v$, or equivalently as the number of edges on the path plus one. We study a refinement of the depth of the external node of rank $s$, with $0 \leq s \leq 2 n$, where the external nodes are ranked/enumerated from left to right according to an inorder-traversal of the tree, by keeping track of three different types of edges on the unique path from the root to the external node. We distinguish between three different types of edges, namely, - left, center, and right-, or equivalently type 1, 2 and 3: an edge $e=(v, w)$, directed from the parent node $v$, being internal, labelled by $\ell(v)$ to node $w$ labelled by $\ell(w)$, is of type $i$ if $\ell(w)=\ell(v)+i-2,1 \leq i \leq 3$. Related parameters have been studied for binary trees in [20, 21, 19]. Moreover, we are interested in the number of embedded ternary trees of size $n$ where all internal nodes have label smaller than or equal to $j$, with $j \in \mathbb{N}$. Furthermore, we study the number of embedded ternary trees of size $n$ counted with respect to the number of internal nodes with label $j$, with $j \in \mathbb{Z}$. We also show how to extend the counting problem to several types of labels considering the nodes with label $j$ and the number of nodes with labels in $\{j-1, j+1\}$, and also discuss generalizations.

\subsection{Plan of the paper}

In the next section we recall some well known properties of the family of ternary trees. In Section 3 we discuss a natural embedding of ternary trees in the plane. Section 4 is devoted to the study of ternary trees with small labels and to ternary trees counted by 
the number of nodes labeled $j$. In Subsection 4.2 we discuss the enumeration problem of counting the number of nodes with label $j$, and at the same time the number of nodes with labels in $\{j-1, j+1\}$. In Section 5 we study the distribution of edge types on the path from the root to the external node of rank $s$ in ternary trees, with $0 \leq s \leq 2 n$. In the final section we discuss some generalizations and open problems, i.e. the extensions of the obtained results to embedded $d$-ary trees.

\section{Preliminaries}

The family of ternary trees $\mathcal{T}$ can be described in a recursive way, which says that a ternary tree is either an external node (a leaf) or an internal node followed by three ordered ternary trees, visually described by the suggestive "equation"

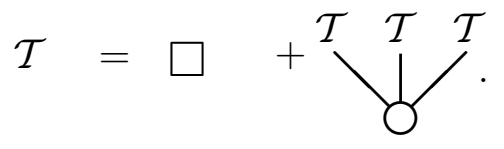

Here $\bigcirc$ is the symbol for an internal node and $\square$ is the symbol for an external node. A trivial consequence of this description is the fact that a ternary tree with $n$ internal nodes has exactly $2 n+1$ external nodes; moreover, by taking external nodes into account any node has either outdegree zero or three. Note that throughout this work the size of the tree is the number of internal nodes, a tree of size $n$ has $n$ internal nodes. We assume that the $2 n+1$ external nodes of a size $n$ ternary tree are numbered from left to right according to a so-called inorder traversal. We start at the root node of a tree. If the tree has internal nodes, we recursively traverse them by going first to the left subtree, then the center subtree, and finally to the right subtree. The external nodes are numbered as visited on the traversal process, see Figure 2. The generating function $T(z)=\sum_{n \geq 0} T_{n} z^{n}$ of the number of ternary trees of size $n$ satisfies the equation

$$
T(z)=1+z T^{3}(z)
$$

Concerning the series expansion of the generating function $T(z)$ it is convenient to consider the shifted series $\tilde{T}(z):=T(z)-1$. The series $\tilde{T}(z)$ satisfies $\frac{\tilde{T}(z)}{(1+\tilde{T}(z))^{3}}=z$. Hence, by the Lagrange inversion formula, see e.g. [14], the number of ternary trees of size $n$ is given by

$$
T_{n}=\left[z^{n}\right] \tilde{T}(z)=\frac{1}{2 n+1}\left(\begin{array}{c}
3 n \\
n
\end{array}\right), \quad n \geq 1 .
$$

Furthermore, we obtain by the Lagrange-Bürmann inversion formula the more general result

$$
\left[z^{n}\right](\tilde{T}(z))^{k}=\frac{k}{n}\left(\begin{array}{c}
3 n \\
n-k
\end{array}\right), \quad \text { for } k \in \mathbb{N}
$$


Note that since $T(z)=\tilde{T}(z)-1$, this immediately implies that for $n \geq k$ we have

$$
\begin{aligned}
{\left[z^{n}\right](T(z))^{k} } & =\left[z^{n}\right](\tilde{T}(z)+1)^{k}=\sum_{\ell=1}^{k}\left(\begin{array}{c}
k \\
\ell
\end{array}\right) \frac{\ell}{n}\left(\begin{array}{c}
3 n \\
n-\ell
\end{array}\right) \\
& =\frac{k}{n}\left(\begin{array}{c}
3 n+k-1 \\
n-1
\end{array}\right)=\frac{k}{3 n+k}\left(\begin{array}{c}
3 n+k \\
n
\end{array}\right) .
\end{aligned}
$$

It turns out that the last representation is valid for all $n \geq 0$. The result above follows by the binomial theorem, Equation (2) and a variant of the Chu-Vandermonde summation formula.

\section{The embedding of ternary trees}

Motivated by the results of Bousquet-Mélou [3], and Panholzer [19] for naturally embedded binary trees, and the results of Schaeffer and Jacquard [13], and Del Lungo, Del Ristoro and Penaud [8] for a subclass of ternary trees, we embed ternary trees in the plane in the following way. An internal node with label/position $j \in \mathbb{Z}$ has exactly three children, being internal or external, placed at positions $j-1, j$ and $j+1$. Following [3], we call this embedding the natural embedding of ternary trees, because the label of a node is its abscissa in the natural integer embedding of the tree. Let $\ell(v)$ denote the label of

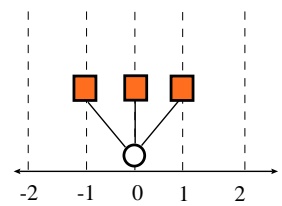

Figure 1: An embedded size 1 ternary tree with its external nodes.

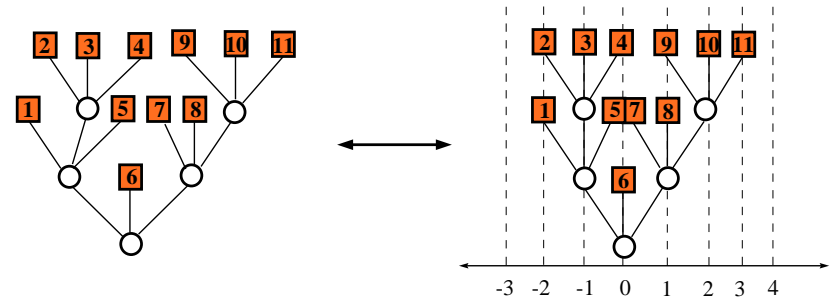

Figure 2: An embedded ternary tree of size 5, its external nodes numbered w. r. t. the inorder-traversal.

node $v \in T$ in a given naturally embedded ternary tree $T$. Due to the labellings of the nodes the edges $e$ of $T$ are decomposed into three different types, types 1, 2 and 3, or equivalently into left, center, and right steps: an edge $e=(v, w)$, directed from the parent node $v$, being internal, to node $w$ is of type $i$, in symbol type $(e)=i$, if $\ell(w)=\ell(v)+i-2$, 


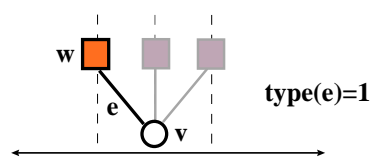

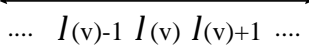

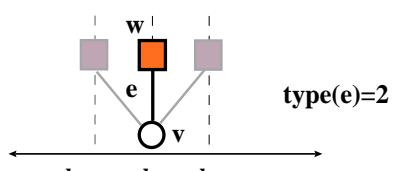

.... l(v)-1 l (v) l(v)+1 $\ldots .$.

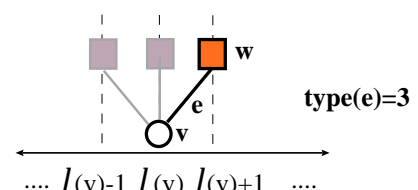

.... l(v)-1 l (v) $l(v)+1 \quad \ldots .$.

Figure 3: The three different edge types.

$1 \leq i \leq 3$; compare with Figure 3 below. Conversely, one may equivalently define the natural embedding solely in terms of three different edge types in the following way. The root of a ternary tree is labelled by zero. Each internal node $v$ has exactly one edge of each type $i, 1 \leq i \leq 3$, pointing away from $v$. The label $\ell(v)$ of a non-root node $v$ in a tree $T$ is then given by the weighted sum $\ell(v)=\sum_{e \in P_{T}(v)}(\operatorname{type}(e)-2)$ over all edges $e$ on the unique path $P_{T}(v)$ from node $v$ to the root of $T$. Note that we associate two different kinds of labels to external nodes, first the label of its position according to the natural embedding, and second the number of the external node according to the inorder-traversal, which we call the rank of the external node. This definition readily extends to $d$-ary trees. It seems natural to decide that for $(2 d)$-ary trees each internal
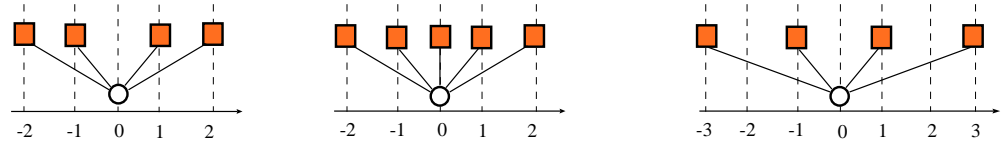

Figure 4: Size one embedded quaternary and quinary trees together with their external nodes; an alternative model for embedded quaternary trees with increments $i \in\{ \pm 1, \pm 3\}$.

node with label $j \in \mathbb{Z}$ has exactly $2 d$ children, internal or external, placed at positions $j+k$, with $k \in\{ \pm 1, \ldots, \pm d\}$, and for $(2 d+1)$-ary trees each internal node with label $j \in \mathbb{Z}$ has exactly $2 d+1$ children, internal or external, placed at positions $j+k$, with $k \in\{0\} \cup\{ \pm 1, \ldots, \pm d\}$; see Figure 4. Equivalently, we have $(2 d)$ or $(2 d+1)$ different types of edges $e_{k}$, with either $k \in\{ \pm 1, \ldots, \pm d\}$ or $k \in\{0\} \cup\{ \pm 1, \ldots, \pm d\}$ which correspond to the steps in the obvious way. We point out that different notions of natural embeddings can be defined. One could alternatively define the embedding of $(2 d)$-ary trees by the increments $k \in\{ \pm 1, \pm 3 \ldots, \pm(1+2 d)\}$, which could be considered more natural than $k \in\{ \pm 1, \ldots, \pm d\}$.

\section{The number of embedded ternary trees with small labels}

Let $T_{j}(z)$ denote the generating function of ternary trees having no internal node of label greater than $j, j \geq 0$, and with $T(z)$ the generating function of ternary trees, as specified by (1). The starting point of our considerations is the following system of equations. 
Lemma 1. The series $T_{j}(z)$ satisfies

$$
T_{j}(z)=1+z T_{j-1}(z) T_{j}(z) T_{j+1}(z), \quad \text { for } j=0,1,2, \ldots, \quad \text { with } T_{-1}(z)=1 .
$$

Proof. First we observe that the infinite system of equations is well defined and completely determines the generating functions $T_{j}(z)$. Moreover, following [3] we note that replacing each label $k$ by $j-k$ shows that the series $T_{j}(z)$ is also the generating function of trees rooted at a node with label $j$, and having only non-negative internal labels. Considering such a tree it has three subtrees rooted at $j+\ell$, with $\ell \in\{-1,0,1\}$, which have again only non-negative internal labels. We have the formal description sketched below in Figure 5, which translates in the stated system of equations and the result follows.

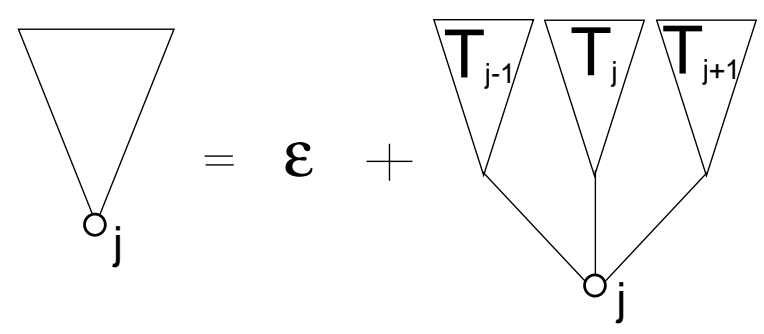

Figure 5: The formal decomposition of embedded ternary trees.

We obtain the following result for the series $T_{j}(z)$.

Theorem 2. Let $T_{j}(z)$ be the generating function of ternary trees with no internal node of label greater than $j$. Then $T_{j}(z)$ is given by the expression

$$
T_{j}(z)=T(z) \frac{\left(1-X^{j+2}(z)\right)\left(1-X^{j+5}(z)\right)}{\left(1-X^{j+3}(z)\right)\left(1-X^{j+4}(z)\right)}, \quad \text { for } j \geq-1,
$$

where $T=T(z)$ is the generating function of ternary trees satisfying $T=1+z T^{3}$, and the series $X=X(z)$ is a formal power series with non-negative coefficients, satisfying $X(0)=0$, and the relation

$$
1-z T^{2}\left(\frac{1}{X}+1+X\right)=0 .
$$

Remark 3. Let $U_{n}(w)$ denote the $n$-th Chebyshev polynomial of the second kind, recursively defined by $U_{0}(w)=1, U_{1}(w)=2 w$ and $U_{n+1}(w)=2 w U_{n}(w)-U_{n-1}(w)$ for $n \geq 1$. Following Bouttier et al. [5] we use the well known closed form expression of $U_{n}(w)$,

$$
U_{n}(w)=\frac{\left(w+\sqrt{w^{2}-1}\right)^{n+1}-\left(w-\sqrt{w^{2}-1}\right)^{n+1}}{2 \sqrt{w^{2}-1}},
$$

and observe that $T_{j}(z)$ can be expressed as the quotient of Chebyshev polynomials of the second kind evaluated at $w=(\sqrt{X}+1 / \sqrt{X}) / 2$, we have for $j \geq 0$ the expression

$$
T_{j}(z)=T(z) \frac{U_{j+1}(w) U_{j+4}(w)}{U_{j+2}(w) U_{j+3}(w)} .
$$


Remark 4. Banderier and Flajolet [1] have studied directed lattice paths in the plane and pointed out the importance of the so-called characteristic polynomial. For so-called simple paths, defined by the set of steps $\mathcal{S}=\left\{\mathbf{a}_{1}, \ldots, \mathbf{a}_{d}\right\}$, with $\mathbf{a}_{\ell}=\left(1, b_{\ell}\right)$ and $b_{\ell} \in \mathbb{Z}$, for $1 \leq \ell \leq d$, the characteristic polynomial is given by $P(X)=\sum_{\ell=1}^{d} X^{b_{\ell}}$, and the characteristic equation of simple paths is given by $1-z P(X)=0$. Note that the equation for the series $X$ for embedded ternary trees can be written as $1-z T^{2}\left(X^{-1}+1+X\right)=0$, which is similar to the characteristic equation of Motzkin path $1-z\left(X^{-1}+1+X\right)=0$. Concerning embedded binary trees [3] the characteristic equation $1-z T(z)\left(X^{-1}+X\right)=0$ is similar to the equation for Dyck path $1-z\left(X^{-1}+X\right)=0$.

Corollary 5. The generating functions $T_{j}(z)$ of ternary trees having no internal node of label greater than $j$, with $j \geq 0$, can be written as fractions in $T=T(z)$, the generating function of ternary trees. In particular, we get for $j=0$ the result

$$
T_{0}(z)=3 T(z)-1-T^{2}(z)
$$

The coefficients $\left[z^{n}\right] T_{0}(z)$ have a simple closed form expression. They are for $n \geq 1$ given by

$$
\left[z^{n}\right] T_{0}(z)=\frac{2}{(n+1)(2 n+1)}\left(\begin{array}{c}
3 n \\
n
\end{array}\right) .
$$

Remark 6. For $j \geq 1$ the coefficients $\left[z^{n}\right] T_{j}(z)$ do not have such simple closed form expression as $\left[z^{n}\right] T_{0}(z)$. Nevertheless, starting from representations like $T_{1}(z)=\frac{(T(z)-2) T^{3}(z)}{T^{2}(z)-3 T(z)+1}$ one readily obtains results similar to

$$
\left[z^{n}\right] T_{1}(z)=\frac{2}{n+1}\left(\begin{array}{c}
3 n \\
n
\end{array}\right)+\sum_{k=0}^{n}(-1)^{k+1} F_{k+1}\left(\begin{array}{c}
3 n \\
n-k
\end{array}\right) \frac{n(11 k+5)-2 k(k+1)}{n(2 n+k+1)}
$$

where $F_{n}=\frac{\phi^{n}-(-1 / \phi)^{n}}{\sqrt{5}}$ denotes the $n$-th Fibonacci number, with $\phi=\frac{1+\sqrt{5}}{2}$.

Remark 7. The result of the case $j=0$ above has already been obtained in $[13,8]$ using bijections with maps and 2-stack sortable permutations. We remark that the sequence of coefficients of $T_{0}(z)$ given by $1,1,2,6,22,91,408, \ldots$ appears as sequence $A 000139$ in the Online Encyclopedia of Integer Sequences [22]. The coefficients of $T_{1}(z)$ given by $1,1,3,11,46,209,1006, \ldots$ are not listed there.

Proof of Theorem 2. In order to proof the result above it is sufficient to check that the series $T_{j}(z)$ satisfies Equation (4) and the initial condition $T_{-1}(z)=1$, which is a simple task. In order to discover the solution we use the method from Bouttier et al. [5], see also Di Francesco [10]. Following [5] we use the fact that for $j$ tending to infinity we have $T_{j}(z) \rightarrow T(z)$. Hence, for $j$ tending to infinity one expects that $T_{j}(z)$ can be written as $T_{j}(z)=T(1+o(1))$. Let the formal power series $\rho_{j}=\rho_{j}(z)$ be defined by $T_{j}(z)=T\left(1-\rho_{j}\right)$, with $\rho_{j} \rightarrow 0$ as $j$ tends to infinity. We rewrite Equation(4) in terms of the series $\rho_{j}$ and obtain

$$
T\left(1-\rho_{j}\right)=1+z T^{3}\left(1-\rho_{j-1}\right)\left(1-\rho_{j}\right)\left(1-\rho_{j+1}\right) .
$$


By definition of the generating function of ternary trees $T=T(z)$ (1) we get further

$$
\rho_{j}=z T^{2}\left(\rho_{j-1}+\rho_{j}+\rho_{j+1}-\rho_{j-1} \rho_{j}-\rho_{j-1} \rho_{j+1}-\rho_{j} \rho_{j+1}+\rho_{j-1} \rho_{j} \rho_{j+1}\right),
$$

with $\rho_{j} \rightarrow 0$ as $j$ tends to infinity. Next we carry out a first order approximation and linearize the recurrence relation for $\rho_{j}$ at large $j$. We introduce the quantity $\hat{\rho}_{j}$, and get the following linear recurrence relation for $\hat{\rho}_{j}$ :

$$
\hat{\rho}_{j}=z T^{2}\left(\hat{\rho}_{j-1}+\hat{\rho}_{j}+\hat{\rho}_{j+1}\right) .
$$

An Ansatz $\hat{\rho}_{j}=\alpha X^{j}$ leads to the so-called characteristic equation

$$
X=z T^{2}\left(1+X+X^{2}\right), \quad \text { or equivalently } \quad 1-z T^{2}\left(X+1+\frac{1}{X}\right)=0 .
$$

Note that the equation above implies several other relations using the relation $z T^{2}=$ $(T-1) / T$; for example

$$
\frac{1}{X}+X=\frac{1-z T^{2}}{z T^{2}}=\frac{1}{T-1} .
$$

Consequently, we define the series $X=X(z)$ as the solution of the characteristic equation above with $X(0)=0$, and a solution of the linearized recurrence relation is given by $\hat{\rho}_{j}=\alpha \cdot X^{j}$. In order to obtain a solution of the original recurrence relation (5) we make the Ansatz $\rho_{j}=\sum_{i \geq 1} \alpha_{i} \cdot\left(X^{j}\right)^{i}$, where $\alpha_{i}=\alpha_{i}(X)$ is independent of $j$, and compare the terms with the same order of magnitude in (5) as $j$ tends to infinity. Note that this formally corresponds to extraction of coefficients of $X^{j i}$ in (5). Using the fact that $1 /\left(z T^{2}\right)-1=X+1 / X$ we obtain the system of recurrences

$$
\begin{aligned}
\alpha_{i+1}\left(X^{i+1}+\frac{1}{X^{i+1}}-X-\frac{1}{X}\right)= & \sum_{\ell=1}^{i} \alpha_{\ell} \alpha_{i+1-\ell}\left(\frac{1}{X^{\ell}}+X^{\ell}+X^{i+1-2 \ell}\right) \\
& -\sum_{\substack{\ell_{1}+\ell_{2}+\ell_{3}=i+1 \\
\ell_{1}, \ell_{2}, \ell_{3} \geq 1}} \alpha_{\ell_{1}} \alpha_{\ell_{2}} \alpha_{\ell_{3}} \frac{X^{\ell_{3}}}{X^{\ell_{1}}}, \quad i \geq 0,
\end{aligned}
$$

which determine $\alpha_{i+1}$ uniquely in terms of $\alpha_{1}$ and rational functions of $X$, with $\alpha_{1}$ unspecified yet. Although it seems at first glance hopeless to obtain a closed form solution, it is possible to guess a solution with the help of computer algebra software, i.e. the help of Maple, which is easily verified using induction. We obtain the surprisingly simple solution

$$
\alpha_{i}=\frac{\alpha_{1}^{i} X^{i-1}\left(1-X^{i}\right)}{(1-X)^{i}\left(1-X^{2}\right)^{i-1}}, \quad i \geq 1 .
$$

We set $\alpha_{1}=\alpha_{1}(X)=\lambda \cdot(1-X)\left(1-X^{2}\right) X^{2}$. Consequently, we get after summation the solution

$$
T_{j}(z, \lambda)=T(z) \frac{\left(1-\lambda X^{j+2}(z)\right)\left(1-\lambda X^{j+5}(z)\right)}{\left(1-\lambda X^{j+3}(z)\right)\left(1-\lambda X^{j+4}(z)\right)}
$$


which satisfies the relation (4) for all $j$, not necessarily larger than zero. In order to see this, we use the notation $v_{j}=1-\lambda X^{j+1}$; the recurrence relation for $T_{j}(z)$, given in Lemma 4, implies that we have to show

$$
T(z) v_{j+1}^{2} v_{j+2} v_{j+3} v_{j+4}^{2}=v_{j+1} v_{j+2}^{2} v_{j+3}^{2} v_{j+4}+z T^{3}(z) v_{j} v_{j+1} v_{j+2} v_{j+3} v_{j+4} v_{j+5},
$$

which is easily seen to be true for all $j \in \mathbb{Z}$, using the relations $z T^{3}=T-1$ and $T=\frac{1+X+X^{2}}{1+X^{2}}$. Finally, adapting to the initial condition $T_{-1}(z)=1$ implies that $\lambda=1$.

Proof of Corollary 5. A simple algebraic proof goes as follows. Let $X$ and $1 / X$ denote the two solutions of the equation $1-z T^{2}\left(\frac{1}{X}+1+X\right)=0$, with $X(0)=0$. The expression

$$
\frac{T_{j}(z)}{T(z)}=\frac{\left(1-X^{j+2}(z)\right)\left(1-X^{j+5}(z)\right)}{\left(1-X^{j+3}(z)\right)\left(1-X^{j+4}(z)\right)}
$$

can be written as a symmetric expression in $X$ and $1 / X$; see also Remark 3 . Since the symmetric functions in $X$ and $1 / X$ are rational functions of $z T^{2}$, and consequently also rational functions of $T$, we obtain the stated result.

Alternatively, one can proceed by induction. Assume that there exist polynomials $p_{\ell}(T)$ and $q_{\ell}(T)$ such that $T_{\ell}=p_{\ell}(T) / q_{\ell}(T)$ can be expressed as a fraction in $T$, for all $-1 \leq \ell \leq j$, with initial values $p_{-1}(T)=1, q_{-1}(T)=1$, and $p_{0}(T)=3 T-1-T^{2}$, $q_{0}(T)=1$. Note that the results for $T_{0}(z)$ are obtained by simple but lengthy calculations. This implies that for $j+1$ we have

$$
T_{j+1}(z)=\frac{T_{j}(z)-1}{z T_{j-1}(z) T_{j}(z)}=\frac{q_{j-1}(T)\left(p_{j}(T)-q_{j}(T)\right) T^{3}}{(T-1) p_{j}(T) p_{j-1}(T)},
$$

such that $p_{j+1}(T)=q_{j-1}(T)\left(p_{j}(T)-q_{j}(T)\right) T^{3}$ and $q_{j+1}(T)=(T-1) p_{j}(T) p_{j-1}(T)$. This proves the stated result. In particular, we obtain for $j=1$ the result

$$
T_{1}(z)=\frac{T_{0}(z)-1}{z T_{-1}(z) T_{0}(z)}=\frac{\left(3 T-2-T^{2}\right) T^{3}}{(T-1)\left(3 T-1-T^{2}\right)}=\frac{(T-2) T^{3}}{T^{2}-3 T+1},
$$

and in general a quick way to easily derive similar results for $T_{2}(z), T_{3}(z)$, etc.

\subsection{The number of vertices with a given label}

Following [3] we are interested in the number of ternary trees of size $n$ with a given number of internal nodes with label $j$. In order to treat this problem we introduce a sequence of bivariate generating functions $S_{j}(z, u)$, where $z$ marks the number of internal nodes, and $u$ the number of internal nodes with label $j$, with $j \in \mathbb{Z}$. Our first observation is already the key point, namely that due to the definition of the embedding of ternary trees, or in other words the symmetry of the step set $\mathcal{S}=\{(1,1),(1,0),(1,-1)\}$, we have the symmetry relation $S_{j}(z, u)=S_{-j}(z, u)$, for all $j \in \mathbb{Z}$; moreover we have $S_{j}(z, 1)=T(z)$. The starting point of our considerations is the following lemma. 
Lemma 8. The series $S_{j}(z, u)$ satisfies

$$
\begin{aligned}
& S_{j}(z, u)=1+z S_{j-1}(z, u) S_{j}(z, u) S_{j+1}(z, u), \quad \text { for } j>0, \\
& S_{0}(z, u)=1+u z S_{-1}(z, u) S_{0}(z, u) S_{1}(z, u)=1+u z S_{0}(z, u) S_{1}^{2}(z, u), \quad \text { for } j=0 .
\end{aligned}
$$

Proof. Using the arguments of [3] we observe that the series $S_{j}(z, u)$ is also the generating function of trees rooted at a node with label $j$, counted by the number of internal nodes labelled zero. The formal description sketched in Figure 5 translates in the stated system of equations and the result follows.

Theorem 9. Let $S_{j}(z, u)$ be the generating function of ternary trees, counted according to the number of internal nodes with label $j$. Then $S_{j}(z, u)$ is given by the the following expression

$$
S_{j}(z, u)=T(z) \frac{\left(1+\mu X^{j+1}(z)\right)\left(1+\mu X^{j+4}(z)\right)}{\left(1+\mu X^{j+2}(z)\right)\left(1+\mu X^{j+3}(z)\right)}, \quad \text { for } j \geq 0,
$$

where $T=T(z)$ is defined by (1), i.e. $T=1+z T^{3}$, the series $X=X(z)$ is specified in Theorem 2, and the power series $\mu=\mu(z, u)$ is defined as the unique formal power series in $z$ satisfying the relation

$$
\mu=(u-1) \frac{(1+\mu X)\left(1+\mu X^{2}\right)^{2}\left(1+\mu X^{5}\right)}{(1+X)^{2}(1-X)^{3}\left(1-\mu^{2} X^{5}\right)} .
$$

The series $\mu(z, u)$ has polynomial coefficients in $u$ and satisfies $\mu(z, 1)=0$.

Proof. We have already seen in the proof of Theorem 9 that the general solution of the set of equations for $j \neq 0$ in Lemma 8 is given by

$$
T_{j}(z, \lambda)=T(z) \frac{\left(1-\lambda X^{j+2}(z)\right)\left(1-\lambda X^{j+5}(z)\right)}{\left(1-\lambda X^{j+3}(z)\right)\left(1-\lambda X^{j+4}(z)\right)} .
$$

We have to determine $\lambda$ in such a way that the equation for $j=0$ in Lemma 8 is satisfied. Using the general solution stated above and the expressions of $T(z)$ and $z$ as functions of $X=X(z)$ we obtain the equation

$$
\begin{aligned}
& \frac{\left(1+X+X^{2}\right)}{\left(1+X^{2}\right)} \frac{\left(1-\lambda X^{2}(z)\right)\left(1-\lambda X^{5}(z)\right)}{\left(1-\lambda X^{3}(z)\right)\left(1-\lambda X^{4}(z)\right)} \\
& \quad=1+u \frac{X}{\left(1+X^{2}\right)} \frac{\left(1-\lambda X^{2}(z)\right)\left(1-\lambda X^{3}(z)\right)\left(1-\lambda X^{6}(z)\right)^{2}}{\left(1-\lambda X^{5}(z)\right)\left(1-\lambda X^{4}(z)\right)^{3}} .
\end{aligned}
$$

Now we simply write $u=(u-1)+1$, and obtain after simple manipulations

$$
-\lambda X=(u-1) \frac{\left(1-\lambda X^{2}\right)\left(1-\lambda X^{3}\right)^{2}\left(1-\lambda X^{6}\right)}{(1+X)^{2}(1-X)^{3}\left(1-\lambda^{2} X^{7}\right)} .
$$

We set $\mu=-\lambda X$, and obtain the stated result. 


\subsection{The number of vertices with given labels}

In this section we derive a generalization of our previous result concerning the enumeration of embedded ternary trees of size $n$ according to the number of internal nodes with label $j$. The crucial fact in the derivation of the previous result was the symmetry relation $S_{j}(z, u)=S_{-j}(z, u)$, which allowed to deduce the equation for the case $j=0$. Hence, when generalizing the counting problem we have to take care to preserve some symmetry in order to set up a system of suitable equations. First, we are interested in counting two statistics at the same time, namely the number of nodes with label $j$, and the number of nodes with label contained in $\{j-1, j+1\}:=\{j \pm 1\}$. Let $S_{j}^{(1)}=S_{j}^{(1)}\left(z, u_{0}, u_{1}\right)$ denote the generating function of ternary trees, where the variable $u_{0}$ counts the number of internal nodes with label $j$, and $u_{1}$ counts the number of internal nodes with labels contained in $\{j \pm 1\}$. Since both types of labels $j-1$ and $j+1$ are counted by the same variable, we have symmetry with respect to the vertical line $j$. More precisely, we obtain the following result.

Lemma 10. The series $S_{j}^{(1)}\left(z, u_{0}, u_{1}\right)$ satisfies the symmetry relation

$$
S_{j}^{(1)}\left(z, u_{0}, u_{1}\right)=S_{-j}^{(1)}\left(z, u_{0}, u_{1}\right)
$$

for all $j \in \mathbb{Z}$. Moreover, $S_{j}^{(1)}=S_{j}^{(1)}\left(z, u_{0}, u_{1}\right)$ is determined by the system of equations

$$
\begin{aligned}
& S_{j}^{(1)}=1+z S_{j-1}^{(1)} S_{j}^{(1)} S_{j+1}^{(1)}, \quad \text { for } j \geq 2, \\
& S_{1}^{(1)}=1+u_{1} z S_{0}^{(1)} S_{1}^{(1)} S_{2}^{(1)}, \quad \text { for } j=1, \\
& S_{0}^{(1)}=1+u_{0} z S_{-1}^{(1)} S_{0}^{(1)} S_{1}^{(1)}=1+u_{0} z S_{0}^{(1)}\left(S_{1}^{(1)}\right)^{2}, \quad \text { for } j=0 .
\end{aligned}
$$

The proof is identical to the proof of Lemma 8 and is therefore omitted. We obtain the following result.

Theorem 11. The generating function $S_{j}^{(1)}\left(z, u_{0}, u_{1}\right)$ of ternary trees, counted according to the number of internal nodes with label $j$ and the number of internal nodes with label in $\{j \pm 1\}$, is given by the expression

$$
S_{j}^{(1)}\left(z, u_{0}, u_{1}\right)=T(z) \frac{\left(1+\nu X^{j}(z)\right)\left(1+\nu X^{j+3}(z)\right)}{\left(1+\nu X^{j+1}(z)\right)\left(1+\nu X^{j+2}(z)\right)}, \quad \text { for } j \geq 1,
$$

where $T=T(z)$ is defined by (1), i.e. $T=1+z T^{3}$, the series $X=X(z)$ is specified in Theorem 2, and the power series $\nu=\nu\left(z, u_{0}, u_{1}\right)$ is defined as the unique formal power series in z satisfying

$$
\begin{aligned}
\nu=\left(u_{0}-1\right) & \frac{X(1+\nu)(1+\nu X)^{2}\left(1+\nu X^{4}\right)}{(1+X)^{2}(1-X)^{3}\left(1-\nu^{2} X^{3}\right)} \\
& +\left(u_{1}-1\right) \frac{\left(1+X^{2}\right)(1+\nu X)\left(1+\nu X^{2}\right)^{3}\left(1+\nu X^{3}\right)}{(1+X)^{2}(1-X)^{3}\left(1-\nu^{2} X^{3}\right)\left(1+\nu X^{4}\right)} .
\end{aligned}
$$


The series $\nu\left(z, u_{0}, u_{1}\right)$ has polynomial coefficients in $u_{1}, u_{2}$ and satisfies $\nu(z, 1,1)=0$. The generating function $S_{0}^{(1)}\left(z, u_{0}, u_{1}\right)$ of ternary trees, counted according to the number of internal nodes with label zero and the number of internal nodes with label in $\{ \pm 1\}$, is given by

$$
S_{0}^{(1)}\left(z, u_{0}, u_{1}\right)=\frac{1}{1-u_{0} z\left(S_{1}^{(1)}\left(z, u_{0}, u_{1}\right)\right)^{2}} .
$$

Remark 12. Note that Theorem 11 is a generalization of our previous result. The series $\nu\left(z, u_{0}, 1\right)$ is related to the series $\mu(z, u)$, stated in Theorem 9 , in the way $\nu(z, u, 1)=$ $X \cdot \mu(z, u)$. Furthermore, the series $S_{j}^{(1)}\left(z, 1, u_{1}\right)$ is the generating function of ternary trees, counted according to the number of internal nodes with label in $\{j \pm 1\}$.

Proof. We proceed similarly to the proof of Theorem 9. The equation for the series $S_{0}^{(1)}$ given in Lemma 10 implies the relation

$$
S_{0}^{(1)}=\frac{1}{1-u_{0} z\left(S_{1}^{(1)}\right)^{2}}
$$

Consequently, we obtain by substituting the result above into the equation for $S_{1}^{(1)}$ the following result:

$$
S_{1}^{(1)}=1+u_{1} z S_{0}^{(1)} S_{1}^{(1)} S_{2}^{(1)}=1+u_{1} z \frac{S_{1}^{(1)} S_{2}^{(1)}}{1-u_{0} z\left(S_{1}^{(1)}\right)^{2}}
$$

Furthermore, we have

$$
S_{1}^{(1)}\left(1-u_{0} z\left(S_{1}^{(1)}\right)^{2}\right)=1-u_{0} z\left(S_{1}^{(1)}\right)^{2}+u_{1} z S_{1}^{(1)} S_{2}^{(1)}
$$

In order to obtain the stated result for $\nu$, we use the general solution

$$
T_{j}(z, \lambda)=T(z) \frac{\left(1-\lambda X^{j+2}(z)\right)\left(1-\lambda X^{j+5}(z)\right)}{\left(1-\lambda X^{j+3}(z)\right)\left(1-\lambda X^{j+4}(z)\right)},
$$

valid for $j>0$, and adapt the parameter $\lambda$ such that the equation between $S_{1}^{(1)}$ and $S_{2}^{(1)}$ is satisfied. Before we actually do so, it is benefical to rewrite $(7)$ by using $u_{1}=\left(u_{1}-1\right)+1$, $u_{2}=\left(u_{2}-1\right)+1$. By simple but lengthy manipulations the equation $(7)$ can be written as

$$
\begin{aligned}
& 1=\left(u_{0}-1\right) \frac{z\left(S_{1}^{(1)}\right)^{2}\left(S_{1}^{(1)}-1\right)}{S_{1}^{(1)}-1-z S_{1}^{(1)}\left(S_{1}^{(1)}\left(S_{1}^{(1)}-1\right)+S_{2}^{(1)}\right)} \\
& \quad+\left(u_{1}-1\right) \frac{z S_{1}^{(1)} S_{2}^{(1)}}{S_{1}^{(1)}-1-z S_{1}^{(1)}\left(S_{1}^{(1)}\left(S_{1}^{(1)}-1\right)+S_{2}^{(1)}\right)}
\end{aligned}
$$


Using the general solution $T_{j}(z, \lambda)$ and the expressions of $T(z)$ and $z$ as functions of $X=X(z)$, we obtain after some simplifications the equation

$$
\begin{aligned}
-\lambda X^{2}=( & \left.u_{0}-1\right) \frac{X\left(1-\lambda X^{2}\right)\left(1-\lambda X^{3}\right)^{2}\left(1-\lambda X^{6}\right)}{(1+X)^{2}(1-X)^{3}\left(1-\lambda^{2} X^{7}\right)} \\
& +\left(u_{1}-1\right) \frac{\left(1+X^{2}\right)\left(1-\lambda X^{3}\right)\left(1-\lambda X^{4}\right)^{3}\left(1-\lambda X^{5}\right)}{(1+X)^{2}(1-X)^{3}\left(1-\lambda^{2} X^{7}\right)\left(1-\lambda X^{6}\right)} .
\end{aligned}
$$

Setting $\nu=-\lambda X^{2}$ leads to the stated result.

Next we briefly discuss the general case. We are interested in the number of nodes with labels in $\{j\}=\{j \pm 0\}$, counted by the variable $u_{0}$, labels in $\{j \pm 1\}$, counted by $u_{1}$, up to labels in $\{j \pm m\}$, counted by $u_{m}$. Note that the cases $m=0$ and $m=1$ correspond to the counting problems treated in Theorems 9, 11. We use the vector notation $\mathbf{u}=\left(u_{0}, \ldots, u_{m}\right)$. Let $S_{j}^{(m)}(z, \mathbf{u})=S_{j}^{(m)}\left(z, u_{0}, \ldots, u_{m}\right)$ denote the generating function of ternary trees, where the variable $u_{\ell}$ counts the number of internal nodes with label in $\{j \pm l\}$, for $0 \leq \ell \leq m$.

Lemma 13. The series $S_{j}^{(m)}=S_{j}^{(m)}(z, \mathbf{u})$ satisfies the symmetry relation $S_{j}^{(m)}=S_{-j}^{(m)}$ for all $j \in \mathbb{Z}$. Moreover, $S_{j}^{(m)}$ is determined by the system of equations

$$
\begin{aligned}
& S_{j}^{(m)}=1+z S_{j-1}^{(m)} S_{j}^{(m)} S_{j+1}^{(m)}, \quad \text { for } j \geq m+1, \\
& S_{j}^{(m)}=1+u_{j} z S_{j-1}^{(m)} S_{j}^{(m)} S_{j+1}^{(m)}, \quad \text { for } 0<j \leq m, \\
& S_{0}^{(m)}=1+u_{0} z S_{-1}^{(m)} S_{0}^{(m)} S_{1}^{(m)}=1+u_{0} z S_{0}^{(m)}\left(S_{1}^{(m)}\right)^{2}, \quad \text { for } j=0 .
\end{aligned}
$$

The equations above are obtained in the same way as the equations in the Lemmata 8,10. However, in the general case $m>1$ it seems more difficult to adapt the generic solution of the set of equations for $j \neq\{-m, \ldots, m\}$ to the $m+1$ equations involving the variables $u_{0}, u_{1}, \ldots, u_{m}$, and to obtain closed form expressions for $S_{j}^{(0)}(z, \mathbf{u}), \ldots, S_{j}^{(m-1)}(z, \mathbf{u})$. In principle one could eliminate the $m$ series $S_{0}^{(m)}, S_{1}^{(m)}, \ldots$, $S_{m-1}^{(m)}$ from the $m+1$ equations to obtain a single equation in $S_{m}^{(m)}$ and $S_{m+1}^{(m)}$, preferably using a computer algebra software, and adapt the generic solution to the single equation. This would then lead to expressions for $S_{j}^{(0)}(z, \mathbf{u}), \ldots, S_{j}^{(m-1)}(z, \mathbf{u})$.

\section{The distribution of edge types}

The depth of a node $v \in T$ is defined as the number of nodes on the unique path $P_{T}(v)$ from the root of the tree $T$ to the node $v$, or equivalently as the number of edges on the path plus one. One can refine the parameter depth of node $v$ by counting the number of edges of type $i, 1 \leq i \leq 3$, on the path from the root of $T$ to node $v$, see Section 3 . We obtain the following result for the distribution of types of edges on the path from the root of the tree to the external node of rank $s, 0 \leq s \leq 2 n$ : 
Theorem 14. The number $T_{n, s, m_{1}, m_{2}, m_{3}}$ of ternary trees of size $n$ where the path from the root to the external node of rank $s$ consists of $m_{i}$ edges of type $i, i=1,2,3$, is for $n \geq 1$ given by the following explicit formulas:

$$
T_{n, s, m_{1}, m_{2}, m_{3}}=\frac{\left(2 m_{3}+m_{2}\right)\left(2 m_{1}+m_{2}\right)\left(\begin{array}{c}
m_{1}+m_{2}+m_{3} \\
m_{1}, m_{2}, m_{3}
\end{array}\right)\left(\begin{array}{c}
3 s_{1}+s_{2}-m_{3}-\mu_{2} \\
s_{1}-m_{3}-\mu_{2}
\end{array}\right)\left(\begin{array}{c}
3 n-m_{1}-2 m_{2}-3 s_{1}+3 u_{2} \\
n-m_{1}-m_{2}-s_{1}+\mu_{2}
\end{array}\right)}{\left(3 s_{1}+s_{2}-m_{3}-\mu_{2}\right)\left(3 n-m_{1}-2 m_{2}-3 s_{1}+3 u_{2}\right)}
$$

for $s=2 s_{1}+s_{2}, 0 \leq s_{1} \leq n$ and $s_{2} \in\{0,1\}$,

$$
T_{n, s, m_{1}, m_{2}, m_{3}}=\frac{2 m_{1}}{3 n-m_{1}}\left(\begin{array}{c}
3 n-m_{1} \\
2 n
\end{array}\right)
$$

for $\left(m_{2}, m_{3}\right)=(0,0), s=0,1 \leq m_{1} \leq n$, and

$$
T_{n, s, m_{1}, m_{2}, m_{3}}=\frac{2 m_{3}}{3 n-m_{3}}\left(\begin{array}{c}
3 n-m_{3} \\
2 n
\end{array}\right)
$$

$$
\text { for }\left(\left(m_{1}, m_{2}\right)=(0,0), s=2 n, 1 \leq m_{3} \leq n \text {. In all other cases } T_{n, s, m_{1}, m_{2}, m_{3}}=0 .\right.
$$

Remark 15. Under the random tree model, i.e. every ternary tree of size $n$ is equally likely, we can consider the random variable $H_{n, s}^{[i]}$ counting the number of edges of type $i$, with $1 \leq i \leq 3$, on the path from the external node of rank $s$ to the root. The joint distribution can be expression in terms of the numbers $T_{n, s, m_{1}, m_{2}, m_{3}}$ and the total number of ternary trees of size $n$,

$$
\mathbb{P}\left\{H_{n, s}^{[1]}=m_{1}, H_{n, j}^{[2]}=m_{2}, H_{n, s}^{[3]}=m_{3}\right\}=\frac{T_{n, s, m_{1}, m_{2}, m_{3}}}{T_{n}} .
$$

Hence, one may derive limiting distribution results in the manner of [19] from the explicit expression for $T_{n, s, m_{1}, m_{2}, m_{3}}$ using Stirling's formula.

Proof. Our methods are based on the considerations of Panholzer [19], see also the works of Panholzer and Prodinger [20,21]. Let $\mathbf{v}=\left(v_{1}, v_{2}, v_{3}\right)$ where the variable $v_{i}$ counts the number of edges of type $i$, with $i=1,2,3$, or equivalently $v_{1}$ counts the "left depth", $v_{2}$ the "center depth", and $v_{3}$ the "right depth". Subsequently we will use the multiindex notation $\mathbf{m}=\left(m_{1}, m_{2}, m_{3}\right)$ and $\mathbf{v}^{\mathbf{m}}=v_{1}^{m_{1}} v_{2}^{m_{2}} v_{3}^{m_{3}}$; moreover $\mathbf{m} \geq 0$ should be interpreted component wise. We introduce the generating function of $T_{n, s, \mathbf{m}}$, the number of ternary trees of size $n$ where the external node of rank $s$ has $m_{i}$ edges of type $i, 1 \leq i \leq 3$, with $1 \leq m_{1}+m_{2}+m_{3} \leq n$,

$$
F(z, u, \mathbf{v})=\sum_{n \geq 0} \sum_{s=0}^{2 n} \sum_{\mathbf{m} \geq 0} T_{n, s, \mathbf{m}} z^{n} u^{s} \mathbf{v}^{\mathbf{m}} .
$$

Note that since we rank the external nodes from left to right, each edge of type $i$ on the unique path from the root of the tree to the considered external node increases the rank of the node by $i-1,1 \leq i \leq 3$. Moreover, each new internal node increases the number 
of external nodes by two. Hence, we get for the external node of rank $s$ the additional condition $s-2 m_{3}-m_{2} \equiv 0 \bmod 2$. The formal description of ternary trees translates into the following functional equation for $F(z, u, \mathbf{v})$, where $T(z)$ denotes the generating function of ternary trees:

$$
\begin{aligned}
F(z, u, \mathbf{v})=1 & +z v_{1} F(z, u, \mathbf{v})(T(z))^{2}+z u v_{2} T\left(z u^{2}\right) F(z, u, \mathbf{v}) T(z) \\
& +z u^{2} v_{3}\left(T\left(z u^{2}\right)\right)^{2} F(z, u, \mathbf{v})
\end{aligned}
$$

We obtain

$$
F(z, u, \mathbf{v})=\frac{1}{1-z v_{1}(T(z))^{2}-z u v_{2} T\left(z u^{2}\right) T(z)-z u^{2} v_{3}\left(T\left(z u^{2}\right)\right)^{2}} .
$$

In order to extract coefficients from $F(z, u, \mathbf{v})$ we use the following folklore result, stated below in full generality.

Lemma 16. The formal power series $1 /\left(1-\sum_{\ell=1}^{k} x_{\ell} \alpha_{\ell}\right)$ in the $k$ variables $x_{1}, \ldots, x_{k}$ satisfies the expansion

$$
\left[x_{k}^{m_{k}} x_{k-1}^{m_{k-1}} \ldots x_{1}^{m_{1}}\right] \frac{1}{1-\sum_{\ell=1}^{k} x_{k} \alpha_{k}}=\left(\begin{array}{c}
m_{1}+\cdots+m_{k} \\
m_{1}, \ldots, m_{k}
\end{array}\right) \prod_{\ell=1}^{k} \alpha_{\ell}^{m_{\ell}} .
$$

Proof. By the multinomial theorem the generating function of $\left(\begin{array}{c}m_{1}+\cdots+m_{k} \\ m_{1}, \ldots, m_{k}\end{array}\right) \prod_{\ell=1}^{k} \alpha_{\ell}^{m_{\ell}}$ is given by $1 /\left(1-\sum_{\ell=1}^{k} x_{\ell} \alpha_{\ell}\right)$, or use

$$
\begin{aligned}
{\left[x_{k}^{m_{k}}\right] \frac{1}{\left(1-\sum_{\ell=1}^{k} x_{\ell} \alpha_{\ell}\right)^{j}} } & =\left[x_{k}^{m_{k}}\right] \frac{1}{\left(1-\sum_{\ell=1}^{k-1} x_{\ell} \alpha_{\ell}\right)^{j}\left(1-\frac{x_{k} \alpha_{k}}{\left(1-\sum_{\ell=1}^{k-1} x_{\ell} \alpha_{\ell}\right)}\right)^{j}} \\
& =\alpha_{k}^{m_{k}} \frac{\left(\begin{array}{c}
m_{k}+j-1 \\
m_{k}
\end{array}\right)}{\left(1-\sum_{\ell=1}^{k-1} x_{\ell} \alpha_{\ell}\right)^{m_{k}+j}}
\end{aligned}
$$

and induction with respect to $k$ concerning the other variables $x_{k-1}, \ldots, x_{1}$.

Consequently, we get for $\left[z^{n} u^{s} \mathbf{v}^{\mathbf{m}}\right] F(z, u, \mathbf{v})$ the result

$$
\begin{aligned}
& {\left[z^{n} u^{s}\right]\left(\begin{array}{c}
m_{1}+m_{2}+m_{3} \\
m_{1}, m_{2}, m_{3}
\end{array}\right) z^{m_{1}+m_{2}+m_{3}} u^{2 m_{3}+m_{2}}\left(T\left(z u^{2}\right)\right)^{2 m_{3}+m_{2}}(T(z))^{2 m_{1}+m_{2}}} \\
& =\left[z^{n-m_{1}-m_{2}-m_{3}} u^{s-2 m_{3}-m_{2}}\right]\left(\begin{array}{c}
m_{1}+m_{2}+m_{3} \\
m_{1}, m_{2}, m_{3}
\end{array}\right)\left(T\left(z u^{2}\right)\right)^{2 m_{3}+m_{2}}(T(z))^{2 m_{1}+m_{2}} .
\end{aligned}
$$

Extraction of coefficients from the powers of $T(z)$ and $T\left(z u^{2}\right)$ will be carried out by an application of our previous result stated in Equation (3); We assume that the rank $s$ of the considered external node, with $0 \leq s \leq 2 n$, is given by $s=2 s_{1}+s_{2}$, for $0 \leq s_{1} \leq n$ and $s_{2} \in\{0,1\}$, with $s_{2}=0$ for $s_{1}=n$. Consequently, we can rewrite the condition 
$s-2 m_{3}-m_{2} \equiv 0 \bmod 2$ into $0 \leq m_{3} \leq s_{1}$ and $m_{2}=s_{2}+2 \mu_{2}$, with $0 \leq \mu_{2} \leq s_{1}-m_{3}$. First we assume that both $\left(m_{1}, m_{2}\right) \neq(0,0)$ and $\left(m_{2}, m_{3}\right) \neq(0,0)$. Using (3) we get

$$
\begin{aligned}
& {\left[z^{n-m_{1}-m_{2}-m_{3}} u^{s-2 m_{3}-m_{2}}\right]\left(T\left(z u^{2}\right)\right)^{2 m_{3}+m_{2}}(T(z))^{2 m_{1}+m_{2}}} \\
& =\frac{\left(2 m_{3}+m_{2}\right)\left(2 m_{1}+m_{2}\right)\left(\begin{array}{c}
3 s_{1}+s_{2}-m_{3}-\mu_{2} \\
s_{1}-m_{3}-\mu_{2}
\end{array}\right)\left(\begin{array}{c}
3 n-m_{1}-2 m_{2}-3 s_{1}+3 u_{2} \\
n-m_{1}-m_{2}-j s_{1}+\mu_{2}
\end{array}\right)}{\left(3 s_{1}+s_{2}-m_{3}-\mu_{2}\right)\left(3 n-m_{1}-2 m_{2}-3 s_{1}+3 \mu_{2}\right)} .
\end{aligned}
$$

Note that we can write the expression above solely in $n, s, m_{1}, m_{2}, m_{3}$,

$$
\begin{aligned}
& {\left[z^{n-m_{1}-m_{2}-m_{3}} u^{s-2 m_{3}-m_{2}}\right]\left(T\left(z u^{2}\right)\right)^{2 m_{3}+m_{2}}(T(z))^{2 m_{1}+m_{2}} }\left(2 m_{3}+m_{2}\right)\left(2 m_{1}+m_{2}\right)\left(\begin{array}{c}
s-m_{3}+\frac{s-m_{2}}{2} \\
\frac{s-m_{2}}{2}-m_{3}
\end{array}\right)\left(\begin{array}{c}
3 n-s-m_{1}-\frac{s+m_{2}}{2} \\
n-m_{1}-\frac{s+m_{2}}{2}
\end{array}\right) \\
&=\frac{\left(s-m_{3}+\frac{s-m_{2}}{2}\right)\left(3 n-s-m_{1}-\frac{s+m_{2}}{2}\right)}{} .
\end{aligned}
$$

Second, we assume that $\left(m_{2}, m_{3}\right)=(0,0)$, which implies that $s=0$ and $1 \leq m_{1} \leq n$. We obtain

$$
\left[z^{n} u^{s} \mathbf{v}^{\mathbf{m}}\right] F(z, u, \mathbf{v})=\left[z^{n-m_{1}}\right](T(z))^{2 m_{1}}=\frac{2 m_{1}}{3 n-m_{1}}\left(\begin{array}{c}
3 n-m_{1} \\
2 n
\end{array}\right) .
$$

Third, let us assume that $\left(m_{1}, m_{2}\right)=(0,0)$, which implies that $s=2 n$ and $1 \leq m_{3} \leq n$. We get

$$
\left[z^{n} u^{s} \mathbf{v}^{\mathbf{m}}\right] F(z, u, \mathbf{v})=\left[z^{n-m_{3}} u^{s-2 m_{3}}\right]\left(T\left(z u^{2}\right)\right)^{2 m_{3}}=\frac{2 m_{3}}{3 n-m_{3}}\left(\begin{array}{c}
3 n-m_{3} \\
2 n
\end{array}\right),
$$

which finishes the proof of Theorem 14 .

\section{Outlook: Embedded d-ary trees}

We discuss generalizations of the results obtained in this note for embedded ternary trees to embedded $d$-ary trees, $d \geq 2$, with respect to the natural embedding of $d$-ary trees defined in Subsection 3.

\subsection{The distribution of edge types}

Subsequently, we will show that the study of the edge types on the path from the root to the external node of rank $s$, with $0 \leq s \leq(d-1) n$, in $d$-ary trees can be treated in the same manner as in the ternary case and binary case. We consider $d$ different types of edges, stemming from the natural embedding. We assume that the $(d-1) n+1$ external nodes of a size $n$ ternary tree are ranked according to an inorder traversal. As before, we introduce the generating function of $T_{n, s, \mathbf{m}}$, the number of $d$-ary trees of size $n$ where path from the root to the external node of rank $s$ consists of $m_{i}$ edges of type $i, 1 \leq i \leq d$, with $1 \leq \sum_{i=1}^{d} m_{i} \leq n$.

$$
F(z, u, \mathbf{v})=\sum_{n \geq 0} \sum_{s=0}^{d n} \sum_{\mathbf{m} \geq 0} T_{n, s, \mathbf{m}} z^{n} u^{s} \mathbf{v}^{\mathbf{m}}
$$


According to the inorder traversal, each edge of type $\ell$ on the unique path from the root to the considered external node increases the rank of the node by $\ell-1$, for $1 \leq \ell \leq d$. Moreover, since each new internal node increases the number of leaves by $d-1$, we obtain the additional condition that for the external node of rank $s$ the numbers $m_{i}$ of edges of type $i, 1 \leq i \leq d$ must satisfy $s-\sum_{\ell=1}^{d} m_{\ell}(\ell-1) \equiv 0 \bmod (d-1)$. The formal description of $d$-ary trees translates into the following functional equation for $F(z, u, \mathbf{v})$, where $T(z)$ denotes the generating function of $d$-ary trees:

$$
F(z, u, \mathbf{v})=1+z \sum_{\ell=1}^{d} v_{\ell} u^{\ell-1}\left(T\left(z u^{d-1}\right)\right)^{\ell-1} F(z, u, \mathbf{v})(T(z))^{d-\ell} .
$$

Consequently, we obtain

$$
F(z, u, \mathbf{v})=\frac{1}{1-z \sum_{\ell=1}^{d} v_{\ell} u^{\ell-1}\left(T\left(z u^{d-1}\right)\right)^{\ell-1}(T(z))^{d-\ell}} .
$$

An application of Lemma 16 to (9) immediately provides

$$
\begin{aligned}
{\left[z^{n} u^{s} \mathbf{v}^{\mathbf{m}}\right] F(z, u, \mathbf{v}) } & =\left(\begin{array}{c}
m_{1}+\cdots+m_{d} \\
m_{1}, \ldots, m_{d}
\end{array}\right)\left[z^{n} u^{s}\right] \prod_{\ell=1}^{d}\left(z u^{\ell-1}\left(T\left(z u^{d-1}\right)\right)^{\ell-1}(T(z))^{d-\ell}\right)^{m_{\ell}} \\
& =\left[z^{n} u^{s}\right] z^{M_{1}} u^{M_{2}-M_{1}}\left(T\left(z u^{d-1}\right)\right)^{M_{2}-M_{1}}(T(z))^{d M_{1}-M_{2}} \\
& =\left[z^{n-M_{1}} u^{s-M_{2}+M_{1}}\right]\left(T\left(z u^{d-1}\right)\right)^{M_{2}-M_{1}}(T(z))^{d M_{1}-M_{2}}
\end{aligned}
$$

where $M_{1}=\sum_{\ell=1}^{d} m_{\ell}$ and $M_{2}=\sum_{\ell=1}^{d} \ell m_{\ell}$. Extraction of coefficients can be carried out via the correspondence to the family of simply generated $d$-ary trees, i.e. the formula

$$
\left[z^{n}\right](T(z))^{k}=\frac{k}{d n+k}\left(\begin{array}{c}
d n+k \\
n
\end{array}\right), \quad \text { for } n \geq 0, k \geq 1, \quad \text { with } T(z)=1+z(T(z))^{d},
$$

where $T(z)$ denotes the generating function of $d$-ary trees. Note that the validity of the equation above for $n \geq 0$ can be shown by using a variant of the Chu-Vandermonde summation formula, compare with (3). Assuming that $1 \leq s \leq(d-1) n-1$ we have

$$
T_{n, s, \mathbf{m}}=\left(\begin{array}{c}
m_{1}+\cdots+m_{d} \\
m_{1}, \ldots, m_{d}
\end{array}\right) \frac{\left(M_{2}-M_{1}\right)\left(d M_{1}-M_{2}\right)\left(\begin{array}{c}
s+\frac{s+M_{1}-M_{2}}{d-1} \\
\frac{s+M_{1}-M_{2}}{d-1}
\end{array}\right)\left(\begin{array}{c}
d n-s-M_{1}+\frac{M_{2}-M_{1}-s}{d-1} \\
n-M_{1}+\frac{M_{2}-M_{1}-s}{d-1}
\end{array}\right)}{\left(s+\frac{s+M_{1}-M_{2}}{d-1}\right)\left(d n-s-M_{1}+\frac{M_{2}-M_{1}-s}{d-1}\right)} .
$$

Assume that $M_{2}-M_{1}=0$, which is equivalent to the condition $s=0$. This implies that $1 \leq m_{1} \leq n$ and $m_{2}=\cdots=m_{d}=0$. We get

$$
\left[z^{n} u^{s} \mathbf{v}^{\mathbf{m}}\right] F(z, u, \mathbf{v})=\left[z^{n-m_{1}}\right](T(z))^{(d-1) m_{1}}=\frac{(d-1) m_{1}}{d n-m_{1}}\left(\begin{array}{c}
d n-m_{1} \\
(d-1) n
\end{array}\right) .
$$

Finally, we consider the case $d M_{1}-M_{2}=0$. This condition is equivalent to $s=(d-1) n$. This implies that $1 \leq m_{d} \leq n$ and $m_{1}=\cdots=m_{d-1}=0$. We obtain the result

$$
\left[z^{n} u^{s} \mathbf{v}^{\mathbf{m}}\right] F(z, u, \mathbf{v})=\left[z^{n-m_{d}} u^{(d-1)\left(n-m_{d}\right)}\right]\left(T\left(z u^{d-1}\right)\right)^{(d-1) m_{d}}=\frac{(d-1) m_{d}}{d n-m_{d}}\left(\begin{array}{l}
d n-m_{d} \\
(d-1) n
\end{array}\right) .
$$




\subsection{Nodes with small labels}

Concerning the enumeration of trees with small labels, the case of $d \geq 4$ is much more difficult than the cases $d=2,3$. One obtains the following system of equations for the generating function $T_{j}(z)$ of $(2 d+1)$-ary trees with no internal node of label greater than $j$ :

$$
T_{j}(z)=1+z \prod_{\ell=-d}^{d} T_{\ell}(z), \quad j \geq 0,
$$

with initial values $T_{j}(z)=1$ for $-d \leq j \leq-1$. The recurrence relations for $(2 d)$-ary trees are identical when skipping the index $\ell=0$ in the product above. In the general case $d \geq 4$ the approach of Bouttier et al. [5] is still applicable, but it is difficult to obtain simple expressions for $T_{j}$. Let the formal power series $\rho_{j}=\rho_{j}(z)$ be defined by $T_{j}(z)=T\left(1-\rho_{j}\right)$, with $\rho_{j} \rightarrow 0$ as $j$ tends to infinity. The linearization of the resulting equation for $\rho_{j}$ leads to a characteristic equation for $X=X(z)$ of the form

$$
1-z T^{2 d}(z) \sum_{\ell=-d}^{d} X^{\ell}=0
$$

For $d \geq 4$ the characteristic equation has more than one solution with $X(0)=0$ and consequently the expression for $\rho_{j}$ gets more involved. See [1] for related problems concerning lattice paths. One can obtain a family of formal power series (in the variable $X=X(z)$ ) for embedded $(2 d+1)$-ary trees,

$$
T_{j}(z)=T \frac{\left(1-\lambda X^{d+1+j}(z)\right)\left(1-\lambda X^{2 d+3+j}(z)\right)}{\left(1-\lambda X^{d+2+j}(z)\right)\left(1-\lambda X^{2 d+2+j}(z)\right)},
$$

where $X$ is a solution of the characteristic equation with $X(0)=0$, and $T=T(z)$ denoting the generating function of $(2 d+1)$-ary trees, $T(z)=1+T^{2 d+1}(z)$, which can be written as a function of $X$. Unfortunately, the stated series can not be used to solve the counting problem; in other words, it is not possible to adapt the series stated above to the initial values $T_{j}(z)=1$ for $-d \leq j \leq-1$. Im the recent work of Bouttier and Guitter [7] a new approach is presented to study equations similar to (10). It may be the key to obtain a simple expression for $T_{j}$. The author is currently investigating into this matter.

\subsection{Some open problems}

We did not manage to derive the generating function of embedded $(2 d+1)$-ary trees for $d>1$. Moreover, we were unable to extend the results for the generating functions of ternary trees to two constraints, i.e. asking for the number of trees with labels only in say $\{0,1, \ldots, m\}, m \geq 0$. We refer the interested reader to the work of Bouttier et al. [6], where a similar problem has been solved for families of plane trees using a $q$-theta function Ansatz, revealing an interesting connection to elliptic functions. 


\section{Acknowledgments}

The author thanks Alois Panholzer for valuable and fruitful discussions concerning ternary trees. Moreover, he thanks Mireille Bousquet-Mélou for encouraging remarks and pointing out several errors. Furthermore, he sincerely thanks the patient anonymous referee, whose very detailed and informative reports led to the correction of numerous imprecisions and errors in the manuscript as well as to an overall improvement of this work.

\section{References}

[1] C. Banderier and P. Flajolet. Basic analytic combinatorics of directed lattice paths. Theoretical Computer Science 281, 37-80, 2002.

[2] M. Bousquet-Mélou and S. Janson, The density of the ISE and local limit laws for embedded trees. Annals Applied Probability, 16, 3, 1597-1632, 2006.

[3] M. Bousquet-Mélou, Limit laws for embedded trees. Applications to the integrated superBrownian excursion. Random Structures and Algorithms 29 , 4, 475-523, 2006.

[4] M. Bousquet-Mélou, Rational and algebraic series in combinatorial enumeration. Proceedings of the ICM. Session lectures, 789-826, 2006.

[5] J. Bouttier, P. Di Francesco and E. Guitter, Geodesic distance in planar graphs, Nucl. Phys. B 663, 535-567, 2003.

[6] J. Bouttier, P. Di Francesco and E. Guitter, Random trees between two walls: Exact partition function, J. Phys. A: Math. Gen. 36 , 12349-12366, 2003.

[7] J. Bouttier and E. Guitter, Planar maps and continued fractions, submitted. Online availaible at http://arxiv.org/abs/1007.0419.

[8] A. Del Lungo, F. Del Ristoro and J.-G. Penaud, Left ternary trees and non-separable rooted planar maps, Theoretical Computer Science 233, 1-2, 201-215, 2000.

[9] L. Devroye and S. Janson, Distances between pairs of vertices and Vertical Profile in conditioned Galton-Watson tree. Random Structures and Algorithms, to appear.

[10] P. Di Francesco, Geodesic Distance in Planar Graphs: An Integrable Approach. The Ramanujan Journal 10, No. 2, 2005.

[11] M. Drmota, Embedded trees and the support of the ISE, Proceedings of the IWOCA 2009 - LNCS 5874, Springer, Berlin, 194-205, 2009.

[12] M. Drmota, Embedded trees and the support of the ISE, submitted.

[13] B. Jacquard and G. Schaeffer, A Bijective Census of Nonseparable Planar Maps, Journal of Combinatorial Theory Series A 83, Issue 1, 1-20, 1998.

[14] I. Goulden and D. Jackson. Combinatorial Enumeration. John Wiley, New York, 1983.

[15] S. Janson, Left and right pathlengths in random binary trees. Algorithmica, 46,419 429, 2006. 
[16] C. Knessl and W. Szpankowski. Binary trees, left and right paths, WKB expansions, and Painléve transcendents. In:Proceedings of the Third Workshop on Analytic Algorithmics and Combinatorics (ANALCO06), Miami, 2006.

[17] J.-F. Marckert, The rotation correspondence is asymptotically a dilatation. Random Structures and Algorithms 24, 118-132, 2004.

[18] A. Meir and J. W. Moon, On the altitude of nodes in random trees. Canadian Journal of Mathematics 30, 997-1015, 1978.

[19] A. Panholzer, Left and right length of paths in Binary trees or on a question of Knuth. Annals of Combinatorics, 12, 479-492, 2009.

[20] A. Panholzer and H. Prodinger, Descendants and ascendants in binary trees. Discrete Mathematics and Theoretical Computer Science, 1, 247-266, 1997.

[21] A. Panholzer and H. Prodinger, Moments of level numbers of leaves in binary trees. Journal of Statistical Planning and Inference, 101, 267-279, 2002.

[22] N. J. A. Sloane, The On-Line Encyclopedia of Integer Sequences (OEIS), Online availaible at http://www.research.att.com/ njas/sequences/, 2011. 